\title{
Forum
}

\section{Neue Bücher zum Psychodrama}

Cossa, M. (2005): Rebels with a Cause: Working with Adolescents Using Action Methods. London, Jessica Kingsley Publishers. ISBN-10: 1-84310-379-6 ISBN-13: 9781843103790

Hale, A., Little, D. $\left(2005^{2}\right)$ : Sociometric Processing of Action Events. The Toronto Centre for Psychodrama and Sociometry. http://www.tcps.on.ca/books.html

Horvatin, T., Schreiber, E. (Hrsg.) (2006): The Quintessential Zerka: Writings by Zerka Toeman Moreno on Psychodrama, Sociometry and Group Psychotherapy. London, Routledge. ISBN 1583917284.

Miller, C. (2006): Souldrama: A Journey Into the Heart of God. Lulu Press. ISBN: 1411696522.

Soppa. P. (2004, 2. überarb. Aufl.): Psychodrama. Ein Leitfaden. Wiesbaden, VS Verlag für Sozialwissenschaften. ISBN 3-8100-4046-0.

\section{Andere interessante Bücher}

Effinger, H. (2006): Lachen erlaubt. Witz und Humor in der Sozialen Arbeit. Regensburg, edition bunte hunde. ISBN 3-934941-12-5.

Neumann, E., Heß, S. (2005): Mit Rollen spielen. Rollenspielsammlung für Trainerinnen und Trainer. Bonn, managerSeminare Verlags GmbH. ISBN 3-936075-35-2.

Spolin, V. (2005): Improvisationstechniken für Pädagogik, Therapie und Theater. Paderborn, Junfermann. ISBN 3-87387-209-9.

Stelzig, M. (2004): Was die Seele glücklich macht. Das Einmaleins der Psychosomatik. Wien, Linde Verlag. ISBN 3-7142-0013-4.

Weinberg, D. (2005): Traumatherapie mit Kindern. Strukturierte Trauma-Intervention und traumabezogene Spieltherapie. Stuttgart, Klett-Cotta. ISBN 3-608-89738-0. 